\title{
Vereine in Deutschland und ihr Beitrag zum Wohlstand der Regionen
}

\author{
Von Axel Franzen und Katrin Botzen
}

Zusammenfassung: Dieser Beitrag untersucht den Zusammenhang zwischen der Anzahl an Vereinen in den 413 Landkreisen und kreisfreien Städten in Deutschland und ihrem wirtschaftlichen und sozialen Wohlstand. Die Analysen basieren auf einer eigenen Vollerhebung des Vereinsbestands bei den für die Vereinsregister zuständigen Amtsgerichten Deutschlands. Zusätzlich verwenden wir die verfügbaren Informationen der statistischen Landes- und Bundesämter zu den Landkreisen und kreisfreien Städten. Als Indikatoren des wirtschaftlichen und sozialen Wohlstands werden Angaben zum Bruttoinlandsprodukt pro Einwohner, der Arbeitslosenquote, der Wahlbeteiligung an der Bundestagswahl 2009 und der Kriminalitätsrate der Regionen genutzt. Für die Zusammenhangsschätzungen werden Spatial-Lag-Regressionen berechnet, mit denen regionale Abhängigkeiten berücksichtigt werden können. Den Ergebnissen zufolge gab es 2008 insgesamt 554.394 eingetragene Vereine in Deutschland, die sich sehr heterogen auf die Regionen verteilen. Die Zusammenhangsanalysen zeigen ferner, dass Kreise mit einer hohen Vereinsdichte auch über ein hohes Bruttoinlandsprodukt pro Einwohner verfügen. Dagegen lassen sich keine statistisch signifikanten Zusammenhänge der Vereinsbestände mit der Arbeitslosenquote, der Wahlbeteiligung oder der Kriminalitätsrate finden.

\section{Einleitung}

Vereine gelten in der Soziologie als ein wichtiges Strukturmerkmal von Gesellschaften. Zwei Klassiker, Alexis de Tocqueville und Max Weber, haben bereits früh auf ihre Bedeutung hingewiesen und dafür plädiert, sie zum Gegenstand sozialwissenschaftlicher Forschung zu machen. So schrieb Alexis de Tocqueville in seinem berühmten Buch über die Demokratie in Amerika: „Meines Erachtens verdient nichts eine größere Aufmerksamkeit als die zu geistigen und sittlichen Zwecken gegründeten Vereine Amerikas“ (Tocqueville [1840] 1976: 599). In Tocquevilles Augen sind Vereine wichtige Bestandteile einer funktionierenden Demokratie. In Vereinen treffen sich Individuen und erhalten Gelegenheiten, ihre Interessen und Meinungen auszutauschen und zu koordinieren. Dies ist eine wichtige Voraussetzung für gemeinschaftliches Handeln. Darüber hinaus lassen sich in Vereinen viele formale Fähigkeiten erlernen. Vereine müssen Satzungen formulieren, Vorsitzende wählen und Treffen organisieren. Dabei üben die Mitglieder sich zu artikulieren und andere von ihren Meinungen zu überzeugen. Vereine werden in Anlehnung an Tocqueville deshalb auch gerne als „Schulen der Demokratie“ bezeichnet (vgl. Almond / Verba 1963).

Auch Max Weber stellte 1910 in seiner bekannten Rede auf dem ersten deutschen Soziologentag die „Soziologie des Vereinswesens“ (Weber 1924: $441 \mathrm{f}$ ) beispielhaft als eine der Aufgaben der Soziologie dar. Vereine stehen nach Weber ,in der Mitte“ der Gesellschaft zwischen der natürlichen Einheit der Familie auf der einen Seite und den offiziellen Organisationen wie Gemeinden, Staat und Kirche auf der anderen Seite. In Webers Worten sind es ,gesellschaftliche Gebilde", denen schon dadurch die Aufmerksamkeit der Soziologie zuteilwerden sollte. Weber interessierte sich unter anderem für die Fragen, wie viele Vereine es in Deutschland gibt, in welche Kategorien sie sich einteilen lassen und ob sie einen vergleichbaren Stellenwert einnehmen wie in Amerika. Er thematisierte ferner die Fragen, in welcher Weise Vereine einerseits Einfluss auf die Persönlichkeit ihrer Mitglieder nehmen und andererseits die Kultur einer Gesellschaft prägen. 
In der modernen sozialwissenschaftlichen Literatur werden Vereine vor allem im Zusammenhang mit dem Begriff Sozialkapital thematisiert. So schreibt etwa der Politologe Robert Putnam: „Networks of civic engagement, like neighbourhood associations, choral societies, cooperatives, sports clubs, mass-based-parties [...] are an essential form of social capital: The denser such networks in a community, the more likely that its citizens will be able to cooperate for mutual benefits“ (Putnam 1993: 173). Ähnliche Formulierungen finden sich auch im deutschen Sprachraum: „Die wichtigste Komponente von Sozialkapital [besteht] im Niveau des tatsächlichen Engagements der Bürger einer Gesellschaft in informellen Netzwerken oder formellen Vereinigungen"(Offe / Fuchs 2001:419). Nach den Vorstellungen der zitierten Autoren entsteht Sozialkapital in Vereinen quasi als Nebenprodukt unabhängig von den konkreten Vereinszielen, indem sich die Akteure in Vereinen treffen und Netzwerke bilden. Diese Netzwerke, so die Argumentation, können zum einen für die beteiligten Akteure von Vorteil sein, etwa durch Informationsaustausch oder durch gegenseitige Hilfeleistungen. Zum anderen entstehen durch die Existenz von Netzwerken auch gesamtgesellschaftliche Vorteile, weil in Gesellschaften mit dichteren Netzwerken die Anreize zu opportunistischem Verhalten reduziert werden.

Vor dem Hintergrund der mutmaßlichen Bedeutung von Vereinen ist es erstaunlich, dass es in Deutschland bisher nur relativ wenige empirische Arbeiten zur Struktur der Vereinslandschaft gibt. Die bestehenden Studien beschränken sich in der Regel auf einzelne Regionen oder Gemeinden oder basieren auf den Angaben von Teilnehmern in Bevölkerungsbefragungen. Da es in Deutschland bisher kein zentrales Vereinsregister gibt, existieren zumeist nur ungenaue Schätzungen zum Vereinsbestand. Die vorliegende Arbeit ist bemüht, einen Beitrag zur Behebung dieser Wissenslücke zu leisten. Wir haben versucht, die Anzahl der Vereine in Deutschland möglichst genau zu erheben. Die gewonnenen Daten erlauben zum einen eine relativ genaue Aussage über den Vereinsbestand in Deutschland. Erstmals kann die Beschreibung für die Ebene der 413 Landkreise in Deutschland vorgenommen werden. Darüber hinaus ermöglichen die Daten auch eine Klassifikation der Vereine, so dass wir detailliert regionale Unterschiede untersuchen können. Neben der Beschreibung des Vereinsbestands nutzen wir die Daten zusätzlich für umfangreiche Zusammenhangsanalysen. Insbesondere fragen wir uns, ob die vermeintlichen Vorteile eines dichten Netzes von Vereinen empirisch nachweisbar sind. Wir untersuchen deshalb, ob Regionen mit einer hohen Vereinsdichte auch die in der Literatur behaupteten Vorteile in Bezug auf ihre wirtschaftliche und soziale Prosperität aufweisen. Im Unterschied zu den bestehenden Studien bestreiten wir unsere Analysen mit prozessproduzierten Daten der regionalen Amtsgerichte und kombinieren diese mit Angaben der statistischen Landes- und Bundesämter. Unsere Analysen weisen damit eine sinnvolle Ergänzung zu den sonst üblichen auf Umfragedaten basierenden Untersuchungen auf.

Der folgende Beitrag ist in vier Abschnitte gegliedert. Im zweiten Kapitel beschreiben wir zunächst kurz den Stand der Forschung zu Vereinen in Deutschland. Dabei gehen wir auf die Fragen ein, was über den Bestand an Vereinen bekannt ist und welche Erkenntnisse über die Auswirkungen von Vereinen theoretisch vermutet und empirisch bestätigt wurden. Am Ende des Kapitels formulieren wir dann eine Reihe von Hypothesen, die die statistischen Zusammenhangsanalysen leiten. Im dritten Abschnitt beschreiben wir unsere Datenerhebung und berichten einige deskriptive Ergebnisse. Mit Hilfe der Daten wird zudem die regionale Verteilung von Vereinen graphisch veranschaulicht. Der vierte Absatz widmet sich der Untersuchung unserer Zusammenhangshypothesen. Wir analysieren dabei die vermuteten Zusammenhänge zwischen dem Vereinsbestand in den Regionen und deren wirtschaftlicher Leistungsfähigkeit. Außerdem interessieren uns weitere Zusammenhänge hinsichtlich einiger sozialer und politischer Indikatoren wie der Kriminalitätsrate und der Wahlbeteiligung. Dabei berücksichtigen wir mittels Spatial-Lag-Regressionen auch die räumliche Abhängigkeit der Daten, die dazu führen kann, dass die Messungen der Eigenschaften der Landkreise nicht 
unabhängig sind. Das letzte Kapitel schließt mit einer Zusammenfassung der wichtigsten Befunde und diskutiert die Vor- und Nachteile unserer Daten.

\section{Zum Stand der Vereinsforschung}

Die empirische Forschung zu Vereinen und zivilem Engagement lässt sich aus methodischer Sicht in zwei Gruppen einteilen. Auf der einen Seite wird mithilfe von Bevölkerungsbefragungen das individuelle Engagement der Bürger erfasst. Bei diesem Ansatz stellen Individuen die Untersuchungseinheiten dar und das primäre Untersuchungsinteresse liegt in der Beschreibung des Unterschieds zwischen dem aktiven und weniger aktiven Teil der Bevölkerung. Auf der anderen Seite können Vereine und Organisationen unmittelbar zum Gegenstand der Forschung gemacht werden. Diese organisationstheoretische Perspektive beschreibt unter anderem die Entwicklung von Anzahl und Typen unterschiedlicher Vereine oder deren Aktivitäten. Für den erstgenannten Ansatz stehen mittlerweile eine ganze Reihe von Bevölkerungsbefragungen zur Verfügung. Alscher et al. (2009) listen 25 Datensätze auf, in denen verschiedene Formen bürgerlichen Engagements in mehr oder weniger regelmäßigen Abständen erfragt werden. Dazu gehören zum Beispiel das Sozioökonomische Panel (SOEP), die Allgemeine Bevölkerungsumfrage der Sozialwissenschaften (ALLBUS), der Freiwilligensurvey oder der Engagementatlas. Entsprechend gibt es eine große Anzahl an empirischen Studien, in denen die eine oder andere Datenquelle für die Analyse der Bestimmungsgründe individuellen Engagements genutzt wird.

Sehr kurz zusammengefasst zeigen diese Studien, dass Mitgliedschaften und Engagement im mittleren Alter (Künemund / Schupp 2007), höheren Bildungsschichten (Erlinghagen 2000; Gensicke et al. 2009: 196ff), in ländlichen Gebieten (Prognos / Generali 2009: 18ff), bei Männern (Gensicke et al. 2006: 13 f; Picot 2001: 15ff), unter Erwerbstätigen und höheren Berufsund Einkommensgruppen (Braun / Klages 2009) und im Westen (van Deth 2001, Freitag / Traunmüller 2008; Jungbauer-Gans 2002; Roth 2001) stärker ausgeprägt sind als bei Frauen, in Städten und den jeweils tieferen Bildungs- und Einkommensgruppen (vgl. Enquete-Kommission 2002 a, 2002 b: 197ff). Personen mit mehr Mitgliedschaften haben darüber hinaus auch mehr Bekannte und Freunde, spenden mehr, sind politisch interessierter und involvierter (Alscher et al. 2009; Priller / Sommerfeld 2009; von Rosenbladt 2009).

Umfragebasierte Daten haben bekanntlich den Nachteil, dass die mit ihnen gewonnenen Erkenntnisse häufig von methodischen Aspekten der Datenerhebung abhängen. So können sich die Art der Stichprobenziehung, die realisierte Ausschöpfungsquote oder die Frageformulierung sehr stark auf die Ergebnisse auswirken. Dies trifft auch auf die umfragebasierte Forschung zum freiwilligen Engagement zu. Nach Priller (2009: 26) variiert die geschätzte Engagementquote je nach verwendeter Datenquelle zwischen 18 (Allensbacher Markt- und Werbeträger-Analyse AWA) und 52 Prozent (Eurobarometer 2006). Es scheint deshalb empfehlenswert, nebst Befragungsdaten auch andere Datenquellen heranzuziehen. Allerdings beschränken sich die bestehenden Studien in Deutschland, in denen Vereine die Untersuchungseinheit bilden, auf bestimmte regionale Gebiete. So liegen zum Beispiel für die Städte Münster und Jena detaillierte Studien über Anzahl, Organisationsstruktur, Finanzierung und Tätigkeitsbereiche der bestehenden Vereine vor (Zimmer 2007). Regionale Vereinsstudien existieren auch für die Städte Mannheim (Maloney et al. 2008) sowie Kassel, München, Zürich (Zimmer et al. 1992) und Hamburg (Horch 1992). Neben diesen regionalen Studien sind häufig Sportvereine Gegenstand der Forschung. Dies hat zwei Gründe: zum einen sind fast alle Sportvereine in Deutschland Mitglied des Deutschen Olympischen Sportbunds e.V. und damit für Erhebungen leichter zugänglich. Zum anderen sind Sportvereine mit schätzungsweise einem Fünftel zahlenmäßig eine der größten Vereinsgruppen. Studien über Sportvereine beschäftigen sich überwiegend mit Finanzierungsquellen und der Mitgliederstruktur (Timm 1979; Heine- 
mann / Schubert 1994; Emrich et al. 2001; Bauer / Braun 2001; Anders 2005; Breuer / Wicker 2010).

In neuerer Zeit wird Vereinen, wie die Zitate aus der Einleitung belegen, in der Diskussion über die mutmaßlichen Vorteile von Sozialkapital ein wichtiger Stellenwert zugeschrieben. Dabei sollte natürlich nicht übersehen werden, dass Vereine lediglich ein Bestandteil einer Komponente von Sozialkapital sind. Unter dem Begriff Sozialkapital werden in der Literatur mindestens drei verschiedene Aspekte zusammengefasst. Zum einen beschreibt der Begriff netzwerkbasierte Ressourcen, auf die die Mitglieder sozialer Netzwerke zugreifen können. Zum anderen bilden aber auch Normen und generalisiertes Vertrauen zwei weitere Komponenten von Sozialkapital (vgl. Franzen / Pointner 2007). In Bezug auf Sozialkapital sind Vereine deshalb wichtig, weil sie ihren Mitgliedern unabhängig vom Vereinszweck und damit gewissermaßen als Nebenprodukt die Möglichkeit bieten, soziale Netzwerke zu bilden und zu pflegen. Gemeinden, Regionen oder Gesellschaften, die eine enge Verknüpfung von Individuen in Netzwerken aufweisen, sollten nach Coleman (1990) und Putnam (1993, 2000) über umfassende Vorteile verfügen. Stärker vernetzte Akteure haben geringere Anreize sich opportunistisch zu verhalten, weil es in ihrem Eigeninteresse liegt, auf ihre Reputation zu achten. Dieser Reputationsmechanismus führt auch dazu, dass sich die Mitglieder in gut vernetzten Gesellschaften stärker vertrauen. Höheres generalisiertes Vertrauen reduziert die Notwendigkeit, wirtschaftliche Aktivitäten vertraglich abzusichern, wodurch die Transaktionskosten geringer werden (Fukuyama 1995). Damit ist zu erwarten, dass Personen mit höherem generalisierten Vertrauen mehr wirtschaftliche Transkationen eingehen und zum Beispiel mehr Dienstleistungen nachfragen. Insgesamt sollte daher in Regionen mit höherem Vertrauen auch eine höhere wirtschaftliche Prosperität vorzufinden sein. Putnam (1993) erklärt die unterschiedliche Wirtschaftsleistung zwischen Süd- und Norditalien mit dem unterschiedlichen Sozialkapitalbestand. Dieser postulierte Zusammenhang zwischen Sozialkapital und Wirtschaftsleistung wurde mittlerweile in einer ganzen Reihe von Studien untersucht. Knack und Keefer (1997) verwenden den World Values Survey (WVS) von 1981 und 1992 und finden für die 29 Länder einen positiven Zusammenhang zwischen generalisiertem Vertrauen und Wirtschaftswachstum. Die erfragte Mitgliedschaft in Vereinen hat dagegen keinen Zusammenhang mit den Wachstumsraten gezeigt. Ähnliche Befunde werden auch von Whiteley (2000) in einer Replikation mit dem WVS berichtet. Beugelsdijk und van Schaik (2005) analysieren die European Values Study (EVS) von 1990 mit 54 Ländern und Regionen. Diesen Ergebnissen zufolge hängt der Anteil aktiver Vereinsmitglieder in einer Region positiv mit dem wirtschaftlichen Wachstum zusammen. Keine robusten Effekte werden hingegen für das generalisierte Vertrauen gefunden. Stadelmann-Steffen und Freitag (2007) finden jedoch in einer Analyse des WVS von 2001, dass das Vertrauen in ärmeren Staaten für das Wirtschaftswachstum förderlich ist. Engagement in Vereinen oder die mit Freunden und Arbeitskollegen verbrachte Zeit spielt dagegen weder in den ärmeren noch in den reicheren Nationen eine Rolle. Insgesamt sind die Ergebnisse damit heterogen, basieren auf kleinen Stichproben von Ländern oder Regionen und beziehen sich auf aggregierte Umfragedaten hinsichtlich der Messung des Sozialkapitals.

Neben dem Wirtschaftswachstum kann Sozialkapital aber auch in Hinsicht auf Arbeitslosenraten relevant sein. Schon Granovetter $(1973,1974)$ hatte argumentiert, dass Personen häufig mithilfe von sozialen Netzwerken nach Arbeitsplätzen suchen. Diese Annahme ist von vielen Studien (siehe für einen Überblick Voss 2007) bestätigt worden. Außerdem lässt sich zeigen, dass die Suche über soziale Netzwerke die Suchdauer nach einem Arbeitsplatz reduziert (Franzen / Hangartner 2006) und eine Analyse des SOEP findet, dass ehrenamtliche Vereinstätigkeit die Arbeitslosigkeit verkürzt (Uhlendorff 2004; Strau $\beta$ 2009). Freitag (2000) vergleicht in einer Studie die Schweizer Kantone und berichtet über geringere Arbeitslosenquoten in Kantonen mit intensiverer Netzwerkeinbindung. Allerdings finden Tolbert et al. (1998) 
keine eindeutigen Zusammenhänge zwischen der Anzahl an Vereinigungen und der Arbeitslosenquote in einer Analyse von 3000 Bezirken in den USA.

Sozialkapital sollte nicht nur in wirtschaftlicher Hinsicht positive Effekte zeitigen. Vielmehr lassen sich in der Literatur viele Hinweise finden, dass Sozialkapital insgesamt die Kooperationsbereitschaft und das Engagement für das Gemeinwohl fördert. Insbesondere wird erwartet, dass Vereinsmitgliedschaften tendenziell das politische Interesse und die politische Partizipation erhöhen. Dementsprechend resümieren schon Almond und Verba (1963: 321) in einer vergleichenden Studie ,the organizational member compared to non-members is likely to consider himself as more competent as a citizen, to be a more active participant in politics, and to know more and care more about politics". Dieser Befund ist in einer ganzen Reihe von Studien immer wieder bestätigt worden. Lippl (2007) hat zum Beispiel den European Social Survey (ESS) von 2002/03 analysiert, der Angaben aus 20 Ländern zu unterschiedlichen Partizipationsformen enthält. Seine Analysen ergeben, dass die Teilnahme an Vereinen auf nahezu alle Indikatoren der politischen Partizipation (unter anderem auf die Wahlbeteiligung) einen positiven Einfluss ausübt (vgl. für ähnliche Befunde auch Kunz / Gabriel 2000).

Die höhere Orientierung am Gemeinwohl durch Sozialkapital sollte des Weiteren eine wichtige Bedeutung bezüglich der Kriminalitätsrate in einer Gesellschaft haben (für einen Überblick siehe Halpern 2005: 113ff). Die Einbettung in soziale Netzwerke erhöht die soziale Kontrolle, womit die Kriminalitätsraten tendenziell geringer ausfallen sollten (Sampson et al. 1997). Putnam (2000) zeigt in bivariaten Zusammenhangsanalysen, dass diejenigen Staaten der USA mit hohem Sozialkapital auch die niedrigsten Kriminalitätsraten aufweisen. Diese Befunde wurden auch durch multivariate Modelle bestätigt. So berichtet Lee (2008) Ergebnisse, die auf der Analyse von über 1.000 ländlichen Bezirken in den USA basieren, dass in Gebieten mit hohem zivilen Engagement (darunter fällt auch die Anzahl an Vereinen pro 1.000 Einwohner) auch tiefere Kriminalitätsraten beobachtet werden (vgl. aber Messner et al. 2004). Ähnliche Ergebnisse liefern Buonanno et al. (2009) für die Häufigkeit von Diebstählen und Raubüberfällen in 103 Regionen Italiens.

Zusammengefasst wird in der Literatur argumentiert, dass Vereine als Bestandteil von Sozialkapital erstens die wirtschaftliche Prosperität und zweitens die Kooperation und die Orientierung am Gemeinwohl fördern. In Bezug auf die wirtschaftliche Prosperität werden dabei in der Regel zwei Indikatoren untersucht: wirtschaftliche Wachstumsraten beziehungsweise das Bruttoinlandsprodukt pro Kopf und die Arbeitslosenquote. Hinsichtlich der Kooperation respektive der Orientierung am Gemeinwohl werden häufig die politische Partizipation, insbesondere die Beteiligung an Wahlen und die Kriminalitätsrate als Indikator verwendet. Auch für die folgenden Analysen verwenden wir diese vier Indikatoren, da sie einerseits für die Landkreise und kreisfreien Städte in Deutschland verfügbar sind und sich anderseits für die Messung des wirtschaftlichen und sozialen Wohlergehens eignen. Unsere vier Hypothesen lassen sich folgendermaßen zusammenfassen:

1. Regionen mit hoher Vereinsdichte sollten über ein höheres Bruttoinlandsprodukt pro Kopf verfügen.

2. Eine höhere regionale Vereinsdichte sollte zu niedrigeren Arbeitslosenquoten führen.

3. Eine höhere Vereinsdichte sollte mit einer höheren politischen Partizipation bzw. Wahlbeteiligung verbunden sein.

4. Regionen mit vielen Vereinen sollten geringere Kriminalitätsraten aufweisen.

Die bestehenden empirischen Untersuchungen zu den vier Hypothesen weisen eine Reihe von Mängeln auf, deren Behebung weitere Untersuchungen rechtfertigen. Erstens sind die vorhandenen Ergebnisse nicht immer eindeutig. Dies trifft insbesondere auf die erste Hypothese zu. Teilweise wird ein Zusammenhang für Assoziationen mit dem Wirtschaftswachstum berichtet, dann wieder scheint lediglich das generelle Vertrauen einen Zusammenhang mit Wirt- 
schaftsdaten aufzuweisen. Zweitens basieren die meisten Untersuchungen auf Umfragedaten, die, wie beispielsweise auch die Ergebnisse für Deutschland zeigen, je nach Datenquelle und Erhebung eine große Spannweite des Engagements ergeben. Drittens basieren die internationalen Vergleiche in der Regel auf kleinen Fallzahlen sehr unterschiedlicher Länder. Hier ist die Gefahr groß, dass die Modelle nicht alle institutionellen oder kulturellen Unterschiede kontrollieren, so dass die Effekte leicht Verzerrungen aufweisen. Viertens beschränken sich diejenigen Studien, in denen Regionen die Analyseeinheiten bilden, zum Teil lediglich auf bestimmte Typen von Regionen (beispielsweise ländliche Gebiete bei Lee 2008) oder verwenden unvollständige Quellen zum Vereinsbestand.

\section{Die Vereinsstruktur in Deutschland}

Aus soziologischer Sicht kann Sozialkapital überall dort entstehen, wo sich Menschen treffen, also natürlich auch in Familien oder Wirtschaftsunternehmungen. Wir konzentrieren uns im Folgenden nur auf den intermediären Sektor, also auf freiwillige Vereinigungen außerhalb des familiären oder wirtschaftlichen Bereichs. Des Weiteren bezieht sich unsere Studie auch nur auf solche Vereinigungen, die eine institutionelle Form und damit einen rechtlichen Status gefunden haben. Dies sind in Deutschland die eingetragenen Vereine. Eine Gruppe von Personen kann den Status ,eingetragener Verein“ von einem Amtsgericht erhalten, sobald sie mindestens sieben Mitglieder sowie einen Vorstand haben und der Vereinszweck nicht auf einen wirtschaftlichen Geschäftsbetrieb gerichtet ist (§21 BGB). Der Verein darf dann das Kürzel „e.V.“ als Bestandteil des Namens führen. Damit bleiben alle informellen Gruppen und Vereinigungen ohne den Zusatz ,e.V.“ in der folgenden Studie unberücksichtigt. Allerdings hat die Anerkennung als ,e.V.“"mehrere Vorteile für Vereinigungen, so dass zumindest größere Vereine Anreize haben, sich eintragen zu lassen. Ein eingetragener Verein ist eine juristische Person. Damit sind Vorsitzende und Mitglieder nicht persönlich bei etwaigen Schäden oder Klagen gegen den Verein haftbar. Zudem kann ein eingetragener Verein Mitarbeiter einstellen, Geschäftskonten führen und Immobilen erwerben. Zusätzlich können eingetragene Vereine vom Finanzamt als ,gemeinnützig“ anerkannt und von der Körperschafts- und Gewerbesteuer befreit werden. Schätzungen zufolge verfügen 80 Prozent der Vereine in Deutschland über diesen Status (Zimmer 2007).

Eingetragene Vereine sind für statistische Untersuchungen sehr viel leichter zugänglich als informelle Gruppen. Allerdings gibt es auch in Deutschland bisher kein vollständiges Zentralregister der Vereine, sondern lediglich regionale Register bei den zuständigen Amtsgerichten. Dieser Umstand erschwert die statistische Erhebung des Bestands an Vereinen erheblich. Wir haben daher im Frühjahr 2008 alle 398 zuständigen Amtsgerichte in Deutschland angeschrieben und um einen Auszug aus dem jeweiligen Vereinsregister gebeten. ${ }^{1}$ Alle Amtsgerichte haben die Gesamtzahl der bei ihnen eingetragenen Vereine mitgeteilt. Aus diesen Angaben ergibt sich, dass im Frühjahr 2008 insgesamt 554.394 eingetragene Vereine in Deutschland registriert waren. Darüber hinaus haben die Amtsgerichte von 483.070 Vereinen den Namen und den Sitz des Vereins mitgeteilt. ${ }^{2}$ Anhand dieser Angaben kann eine Klassifizierung der Vereine nach Vereinstyp vorgenommen werden. Zusätzlich lassen sich die Vereine den 413 Landkreisen und kreisfreien Städten in Deutschland zuordnen, so dass erstmals die

1 Die Erhebung wurde in Zusammenarbeit mit V\&M Service GmbH in Konstanz durchgeführt. Für einen ersten deskriptiven Ergebnisbericht siehe Happes (2008) und Franzen / Botzen (2009).

2 Von den restlichen 71.324 Vereinen liegen uns keine Vereinsnamen vor, weil einige Amtsgerichte die Vereinsregister noch mittels Karteikarten führten. Die Angaben hätten in diesen Fällen mit erheblichem Aufwand übertragen werden müssen. 
Anzahl der Vereine nach Vereinstyp auf Landkreisebene dargestellt werden kann. ${ }^{3}$ Die Daten ermöglichen damit die Beschreibung der regionalen Verteilung von Vereinen in Deutschland - womit sich eine „Landkarte“ der Vereinsdichte erstellen lässt.

Vergleicht man die Bundesländer (Tabelle 1), so hat Nordrhein-Westfalen mit 115.190 absolut gesehen die meisten Vereine und Bremen und Hamburg mit 3.282 und 9.077 die geringste Anzahl an Vereinen. Im Verhältnis zur Einwohnerzahl führt das Saarland mit 9 Vereinen auf 1.000 Einwohner die Liste der Bundesländer an. Bayern, Nordrhein-Westfalen und Niedersachsen liegen hier unter dem deutschen Mittelwert von 6,77 pro 1.000 Bürger. Das Schlusslicht bildet der Stadtstaat Hamburg mit 5,1 Vereinen und Schleswig-Holstein mit 5,46 Vereinen pro 1.000 Einwohner.

Die Vereinsdichte lässt sich auch detaillierter für die 413 Landkreise und kreisfreien Städte darstellen. Zur Veranschaulichung haben wir die Anzahl der Vereine pro 1.000 Einwohner in eine Deutschlandkarte übertragen. Die dunklen Flächen in der Landkarte weisen auf eine hohe Anzahl an Vereinen pro Einwohner hin. Mit zunehmender Helligkeit der Einfärbungen wird die Zahl der eingetragenen Vereine pro Einwohner geringer (Abbildung 1).

Tabelle 1: Vereine in den Bundesländern Deutschlands

\begin{tabular}{lrrcc}
\hline \hline Bundesland & Vereine & $\%$ & pro 1.000 Einw. & Verein: Einw. \\
\hline Saarland & 9.338 & 1,7 & 9,00 & $1: 111$ \\
Thüringen & 19.866 & 3,6 & 8,65 & $1: 115$ \\
Rheinland-Pfalz & 32.760 & 5,9 & 8,04 & $1: 123$ \\
Baden-Württemberg & 83.107 & 15,0 & 7,72 & $1: 129$ \\
Mecklenburg-Vorpommern & 12.516 & 2,3 & 7,43 & $1: 134$ \\
Hessen & 42.645 & 7,7 & 7,00 & $1: 142$ \\
Sachsen-Anhalt & 16.820 & 3,0 & 6,93 & $1: 143$ \\
Sachsen & 28.809 & 5,2 & 6,81 & $1: 146$ \\
Bayern & 81.526 & 14,7 & 6,48 & $1: 154$ \\
Nordrhein-Westfalen & 115.190 & 20,8 & 6,38 & $1: 156$ \\
Niedersachsen & 48.974 & 8,8 & 6,12 & $1: 163$ \\
Berlin & 20.156 & 3,6 & 5,88 & $1: 169$ \\
Bremen & 3.828 & 0,7 & 5,68 & $1: 173$ \\
Brandenburg & 14.247 & 2,6 & 5,61 & $1: 178$ \\
Schleswig-Holstein & 15.535 & 2,8 & 5,46 & $1: 183$ \\
Hamburg & 9.077 & 1,6 & 5,10 & $1: 195$ \\
\hline Deutschland & 554.394 & 100,0 & 6,77 & $1: 148$ \\
\hline \hline
\end{tabular}

3 Die Zuordnung auf Vereinsarten bei den restlichen 71.324 Vereinen, deren Vereinsname von den Amtsgerichten nicht berichtet werden konnte, haben wir aufgrund der Verteilung in den umliegenden Landkreisen geschätzt. Diese Schätzung betrifft aber nur die Verteilung auf Vereinsarten und nicht die Anzahl der Vereine, die von jedem Landkreis bekannt ist. 
Abbildung 1: Eine Landkarte der Vereinsdichte

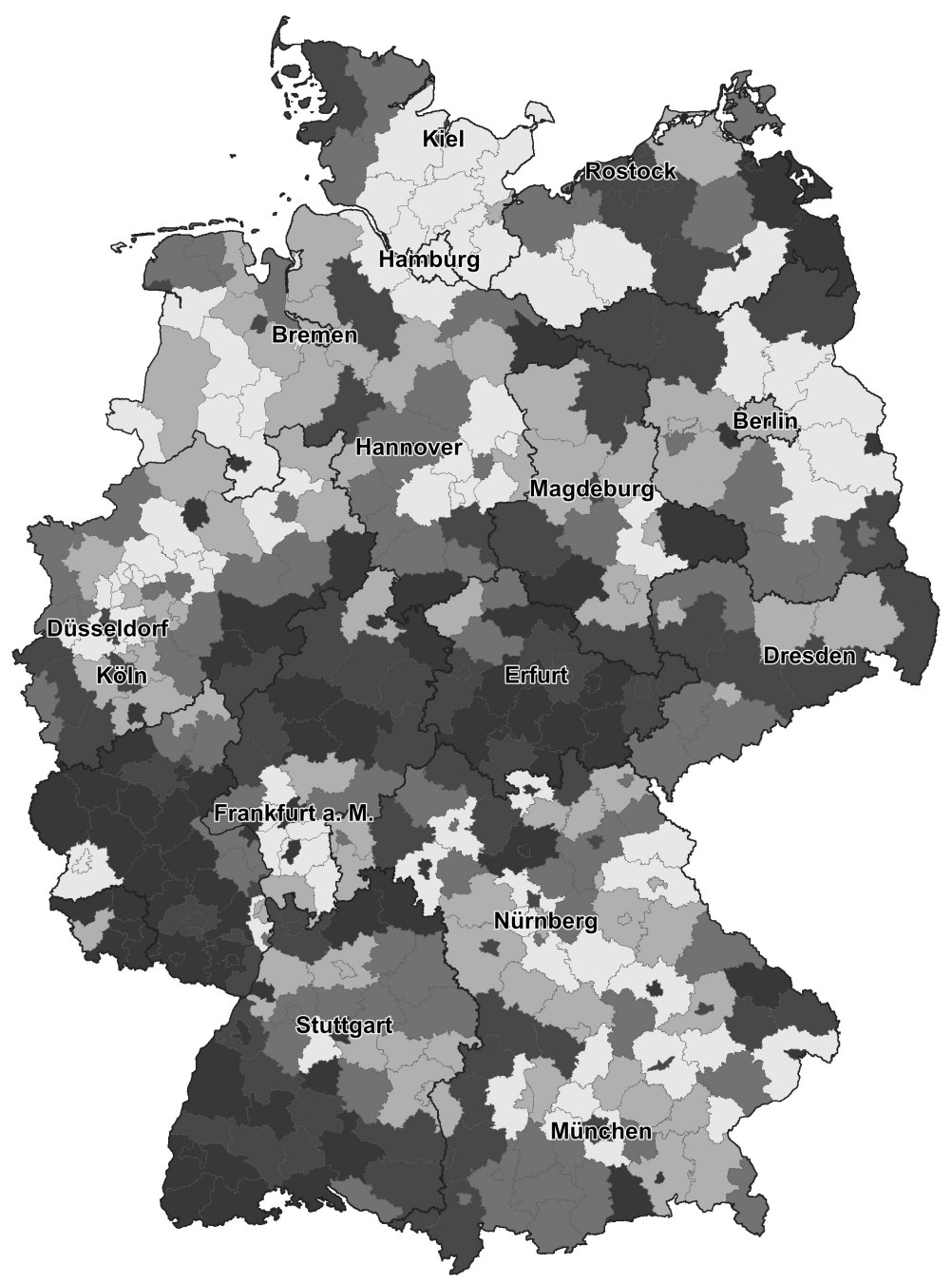

Gesamtzahl der Vereine auf 10.000 Einwohner Stadt- und Landkreise Deutschland

$$
\begin{array}{r}
\leq 54.20 \\
\leq 62.45 \\
\leq 70.38 \\
\leq 82.44
\end{array}
$$

Der Abbildung 1 lässt sich entnehmen, dass die Vereine in Deutschland sehr heterogen verteilt sind. Es ist weder ein Ost-West Gefälle, noch ein Nord-Süd Gefälle zu erkennen. Eine hohe Dichte an Vereinen ist im Süden von Baden-Württemberg, in nahezu ganz Rheinland-Pfalz und in Teilen von Hessen und Thüringen zu erkennen. Zudem gibt es punktuell in ganz Deutschland hohe Konzentrationen an Vereinen: Besonders in dem Stadtkreis Karlsruhe mit 17,3 Vereinen sowie in der Region Bamberg mit 15 Vereinen pro 1.000 Einwohner. Über- 
durchschnittlich viele Vereine gibt es auch in der kreisfreien Stadt Münster (11,6 Vereine) und im Landkreis Kusel (Rheinland-Pfalz, 12,1 Vereine). Die geringsten Konzentrationen an Vereinen haben der Odenwaldkreis (Hessen) und der Landkreis Oder-Spree (Brandenburg) mit jeweils einem Verein, gefolgt von der kreisfreien Stadt Oberhausen, in der nur zwei Vereine pro 1.000 Einwohner gezählt wurden.

In einem weiteren Schritt lassen sich die Vereine anhand der Vereinsnamen nach dem mutmaßlichen Vereinszweck klassifizieren. Diese Klassifikation war wegen der großen Anzahl an Vereinen mit einem erheblichen Aufwand verbunden. Des Weiteren konnte nicht immer aus den Vereinsnamen ein eindeutiger Vereinszweck herausgelesen werden. In diesen Fällen wurde der Verein im Internet recherchiert und eine Zuordnung anhand der Vereinssatzung vorgenommen. Wir haben die Vereine in sieben Gruppen eingeteilt: (1) Umwelt/Natur, (2) Kultur, (3) Sport, (4) Interessen, (5) Wirtschaft/Politik, (6) Soziales/Wohlfahrt und (7) Freizeit. ${ }^{4}$ Diese Einteilung orientiert sich an der Klassifikation des Center for Civil Society Studies der Johns Hopkins University. Um die Darstellungen der Verteilung auf eine überschaubare Anzahl zu beschränken (siehe Abbildungen 2 a bis 2 c), wurden die Vereinskategorien durch eine explorative Faktorenanalyse (Varimax-Rotation) von sieben Kategorien auf drei Vereinsgruppen reduziert. 80 Prozent der Varianz der Vereine werden durch drei Faktoren erklärt. Dies sind erstens Natur-, Kultur- und Sportvereine, zweitens Politik-, Sozial- und Interessensvereine und drittens Freizeitvereine. Die Anzahl der Vereine in den beiden ersten Vereinstypen wurde dann jeweils zu einem Index aufsummiert.

Tabelle 2: Vereinskategorien und deren Faktoren

\begin{tabular}{|c|c|c|c|c|c|c|}
\hline Rubrik & Faktor & Vereine & $\%$ & $\begin{array}{c}\text { pro } 1.000 \\
\text { Einw. }\end{array}$ & $\begin{array}{l}\text { erklärte } \\
\text { Varianz }\end{array}$ & Eigenwert \\
\hline Umwelt/Natur & & 7.477 & 1,3 & 0,09 & 33,67 & 3,05 \\
\hline Kultur & 1 & 28.952 & 5,2 & 0,35 & & \\
\hline Sport & & 95.990 & 17,3 & 1,17 & & \\
\hline Interessen & & 46.231 & 8,3 & 0,56 & 27,46 & 1,45 \\
\hline Wirtschaft/Politik & 2 & 84.731 & 15,3 & 1,03 & & \\
\hline Soziales/Wohlfahrt & & 100.689 & 18,2 & 1,23 & & \\
\hline Freizeit & 3 & 188.144 & 33,9 & 2,29 & 18,03 & 1,04 \\
\hline Gesamt & & 554.394 & 100,0 & 6,74 & 79,16 & \\
\hline
\end{tabular}

Die „Landkarten“ der beiden Indizes und der Freizeitvereine sind sehr unterschiedlich und auch durch die Differenzierung ist kein eindeutiges Ost-West- oder Nord-Süd-Gefälle zu erkennen (siehe Abbildung $2 \mathrm{a}-2 \mathrm{c}$ ). Es fällt auf, dass in Baden-Württemberg und Thüringen eine hohe Konzentration an Natur-, Kultur- und Sportvereinen besteht und in Rheinland-Pfalz und Sachsen-Anhalt überdurchschnittlich viele Freizeitvereine existieren. Ansonsten erscheint die Verteilung von Vereinen sehr heterogen zu sein. Viele Vereine gibt es vor allem in den kreisfreien Städten. Die kreisfreien Städte Münster und Bonn liegen mit 7,2 und 8,7 Politik-, Sozial- und Interessensvereinen pro 1.000 Einwohner an der Spitze dieser Vereinstypen. Die Natur-, Kultur- und Sportvereine sind in Deutschland in den kreisfreien Städten Baden-Baden und Karlsruhe (Baden-Württemberg) mit 5,4 und 8,1 am stärksten vertreten.

4 In den meisten Fällen ist die Zuordnung von Vereinen in diese Kategorien unproblematisch, weil bei vielen Vereinen der Vereinszweck im Namen ersichtlich ist. Beispielsweise fällt der „Sport-Verein Godorf 1956 e.V.“ eindeutig in die Kategorie Sport oder der „Stammdesch Kölsche Klüngel e.V.“ in die Kategorie Freizeit. Schwieriger ist die Zuordnung bei Vereinen mit mehrdeutigen Namen. Beim „Natur- und Wanderfreunde Nassau e.V.“ liegt der Hauptzweck z.B. nicht im Naturschutz, sondern im gemeinsamen Wandern. Es handelt sich daher um einen Freizeitverein. 
Für die Freizeitvereine lässt sich beobachten, dass die höchsten Konzentrationen in Landkreisen und nicht in kreisfreien Städten zu finden sind. Der Landkreis Uecker-Randow in Mecklenburg-Vorpommern verzeichnet mit 6,2 Freizeitvereinen pro 1.000 Einwohner die größte Anzahl dieser Vereinsart. Die Landkreise Bamberg (Bayern) und Kusel (RheinlandPfalz) zeigen mit 5,8 und 5,6 Freizeitvereinen ebenfalls überdurchschnittlich viele Freizeitvereine pro 1.000 Einwohner auf. Über die drei Vereinshauptkomponenten hinweg, lässt sich feststellen, dass die Landkreise Oder-Spree, Märkisch-Oderland und Dahme-Spreewald (alle in Brandenburg gelegen) und der Odenwaldkreis in Hessen die geringste Dichte an Vereinen aufweisen. Auch der Landkreis Herford und die kreisfreie Stadt Oberhausen sind unter den sieben Regionen Deutschlands mit der niedrigsten Anzahl an Vereinen. 
Abbildung 2 a: Die Verteilung von Natur-, Kultur- und Sportvereinen

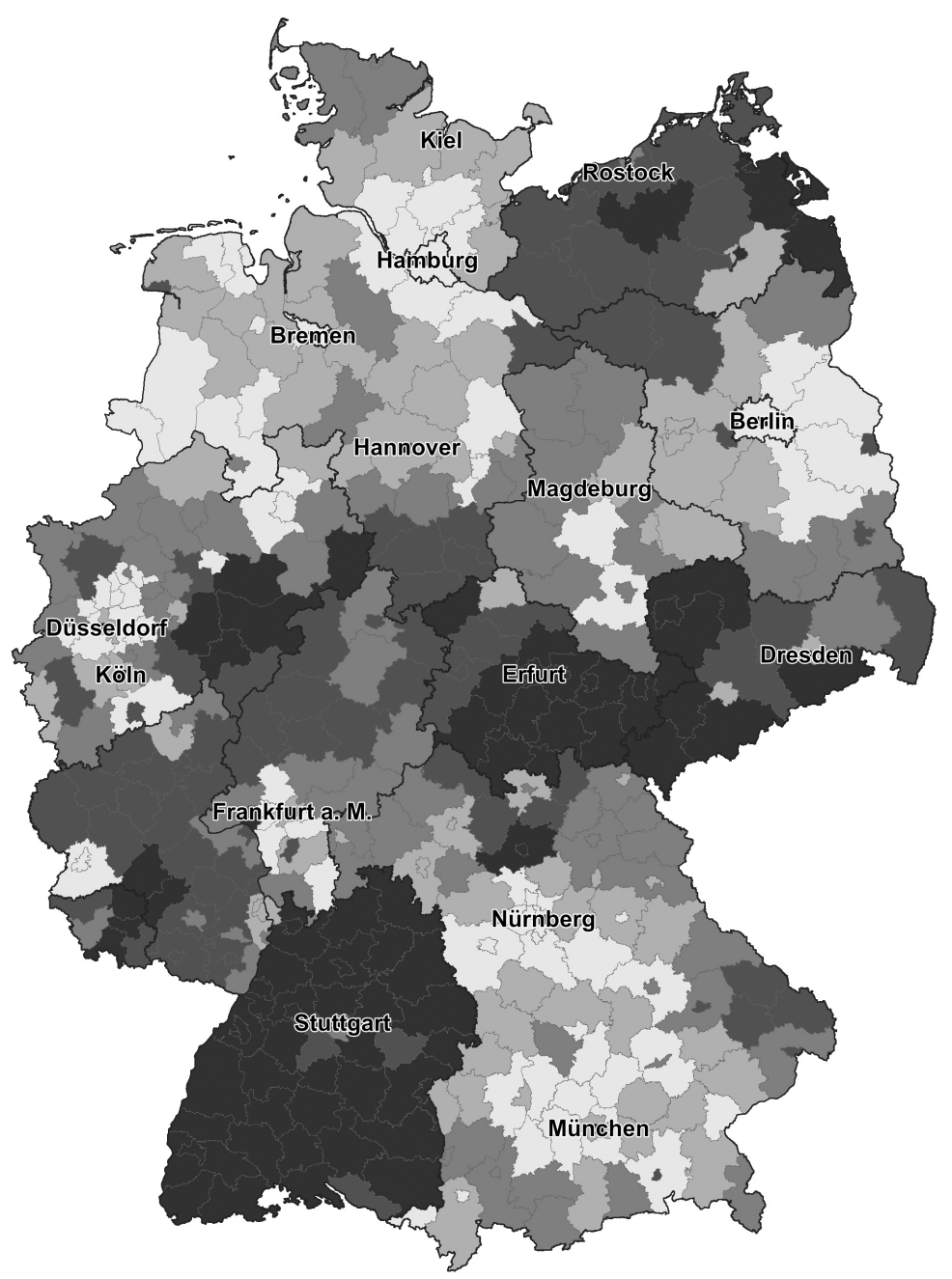

Natur-, Kultur- und Sportvereine 10.000 Einwohner Stadt- und Landkreise Deutschland

$$
\begin{aligned}
& \leq 54.20 \\
\leq & 62.45 \\
\leq & 70.38 \\
& \leq 82.44 \\
& \leq 200.0
\end{aligned}
$$


Abbildung 2 b: Die Verteilung von Politik-, Sozial- und Interessensvereinen

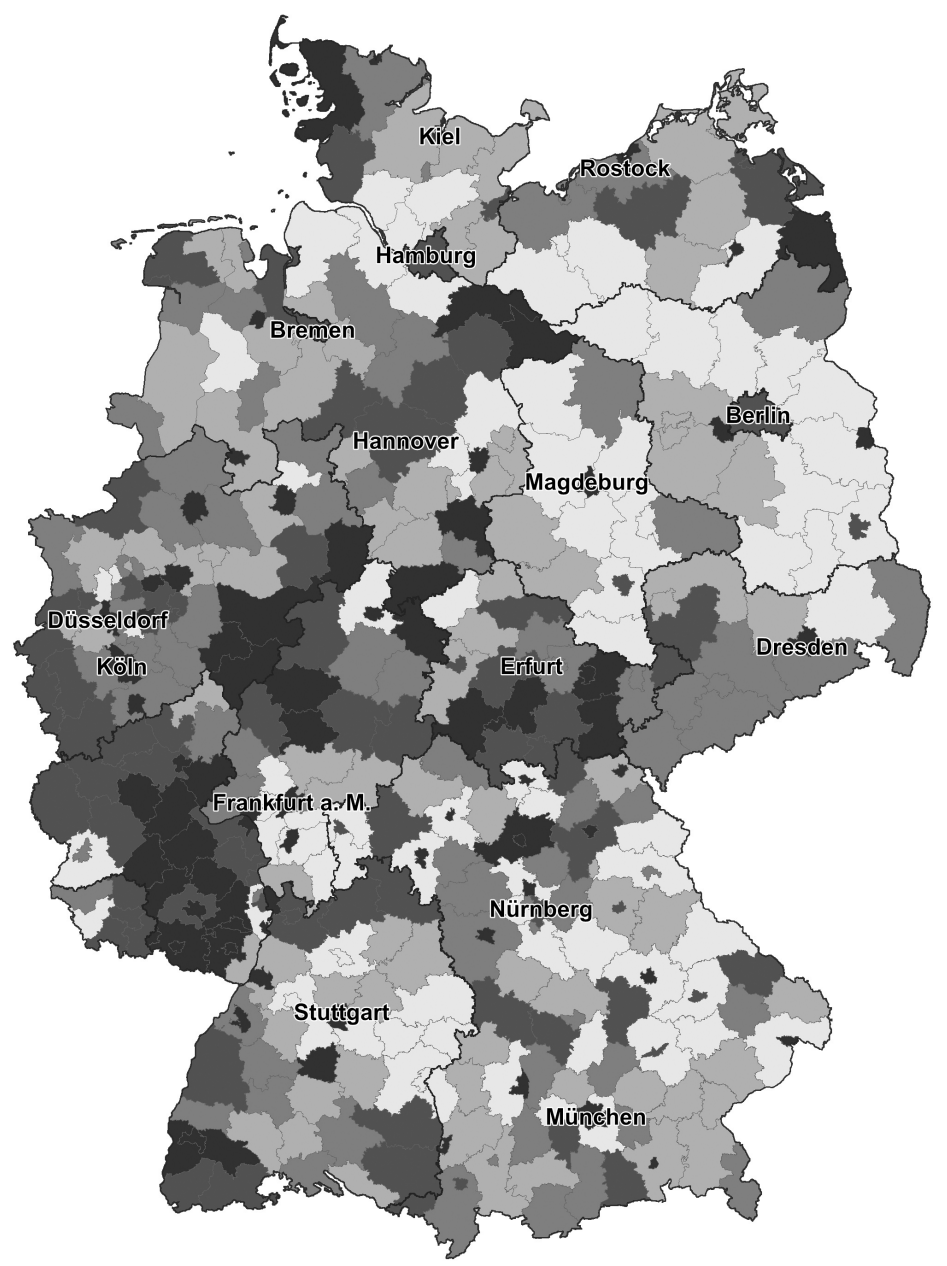

Sozial-, Interessens- und Politikvereine 10.000 Einwohner Stadt- und Landkreise Deutschland

$$
\begin{aligned}
& \leq 54.20 \\
& \leq 62.45 \\
& \leq 70.38 \\
& \leq 82.44 \\
& \leq 200.0
\end{aligned}
$$


Abbildung 2c: Die Verteilung von Freizeitvereinen

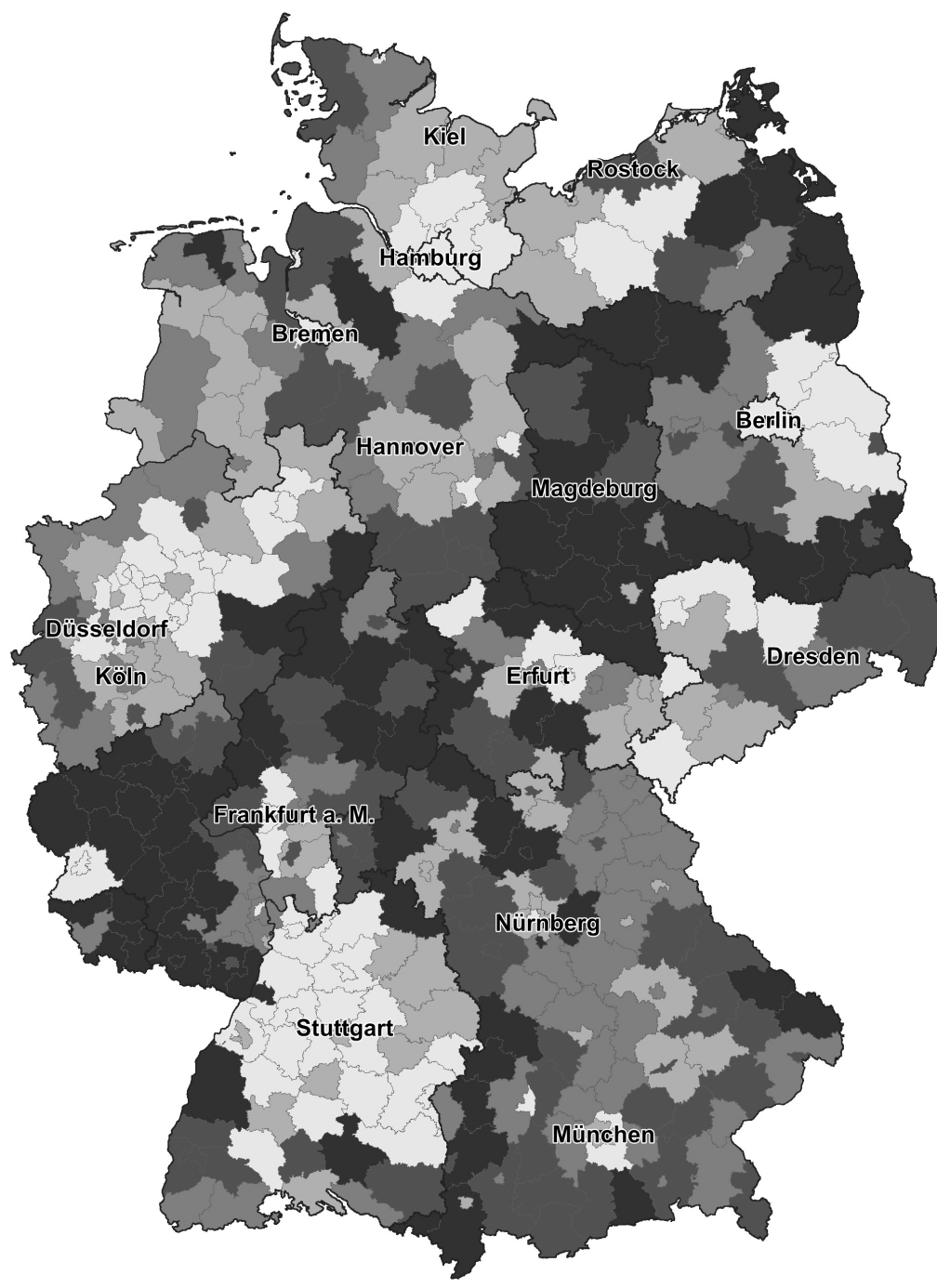

Freizeitvereine auf 10.000 Einwohner

Stadt- und Landkreise Deutschland

$$
\begin{aligned}
& \leq 54.20 \\
& \leq 62.45 \\
& \leq 70.38 \\
& \leq 82.44 \\
& \leq 200.0
\end{aligned}
$$

(C) Für die Abbildungen wurde RegioGraph (GfK Geomarketing) verwendet. 


\section{Vereine und der Zusammenhang mit wirtschaftlichem und sozialem Wohlstand}

Die Vereinsdaten lassen sich für die empirische Untersuchung der formulierten Hypothesen zum positiven Einfluss von Vereinen für Regionen nutzen. Zu diesem Zweck haben wir die von den statistischen Landes- und Bundesämtern verfügbaren und relevanten Informationen über Landkreise und kreisfreie Städte mit der Vereinsstatistik verbunden. Als Indikatoren für die wirtschaftliche Prosperität verwenden wir das Bruttoinlandsprodukt pro Kopf und die Arbeitslosenquote der Landkreise für 2009 als abhängige Variable. Unsere sozialen Wohlfahrtsindikatoren sind die Beteiligung an der Bundestagswahl und die Kriminalitätsrate ebenfalls beides für das Jahr 2009. Alle vier Variablen weisen erhebliche Varianzen auf (siehe Anhang A1). Der ärmste Landkreis ist der Landkreis Südwestpfalz (15.055 Euro BIP pro Einwohner) und der Landkreis München ist mit Abstand die reichste Region in Deutschland (91.860 Euro BIP pro Kopf). Im Landkreis Eichstätt in Bayern betrug die Arbeitslosenquote nur 2,5 Prozent und im Landkreis Demmin (Mecklenburg-Vorpommern) 20,1 Prozent. Im Würzburg (Bayern) wird mit 26 Straftaten pro 1.000 Einwohner und Jahr die geringste Delinquenz registriert, hingegen sind es in Parchim inklusive der Stadt Schwerin 209 Straftaten. Die höchste Wahlbeteiligung findet sich im Hochtaunuskreis (Hessen) mit 80 Prozent und die niedrigste liegt im Salzlandkreis (Sachsen-Anhalt) bei 56,6 Prozent.

Als unabhängige Kontrollvariablen verwenden wir alle verfügbaren Angaben über die Landkreise, die einen Einfluss auf die vier Indikatoren haben könnten. Dazu gehört die Wirtschaftstruktur (Anteil des Dienstleistungssektors), die Bildungsbeteiligung (Anteil an Schulabgängern mit Studienzugangsberechtigung), die Bevölkerungsdichte (Einwohner pro 100 $\mathrm{km}^{2}$ ), die Sozialhilfequote (Anteil an Personen, die staatliche Transfereinkommen beziehen), der Ausländeranteil, die Altersstruktur und die Sex-Ratio in den Landkreisen. Zusätzlich enthält die rechte Seite der OLS-Gleichung 12 Dummy-Variablen für die Bundesländer (Referenz ist Nordrhein-Westfalen), eine weitere Dummy-Variable für Großstädte (Städte über 100.000 Einwohner, hier sind auch die Stadtländer Hamburg, Bremen und Berlin enthalten) und eine Dummy-Variable um Landkreise von kreisfreien Städten zu separieren. Die wirtschaftliche und soziale Prosperität kann auch davon abhängen, ob die Landkreise eine Grenze zu einem Nachbarstaat aufweisen oder nicht. Aus diesem Grund enthalten die Analysen eine weitere Dummy-Variable, mit der Landkreise mit und ohne ausländische Grenze unterschieden werden.

In Studien, in denen Regionen die Analyseeinheiten bilden, treten in der Regel regionale Abhängigkeiten bei der Messung der zu erklärenden Merkmale auf. Beispielsweise kann das Bruttoinlandsprodukt eines Landkreises durch das Bruttoinlandsprodukt der umliegenden Landkreise beeinflusst werden. Damit würde bei Verwendung einfacher OLS-Regression die Bedingung der unabhängigen Messung der zu erklärenden Merkmale verletzt (Anselin 1988, LeSage / Pace 2009, Ward / Gleditsch 2008). Eine solche Autokorrelation lässt sich mithilfe sogenannter Spatial-Error- (Tolbert et al. 1998) oder Spatial-Lag-Modelle berücksichtigen (vgl. Buonanno et al. 2009; Chi / Zhu 2008). Wir verwenden in unseren Analysen Spatial-LagModelle. Zu diesem Zweck haben wir für jeden Landkreis die Anzahl angrenzender Landkreise ermittelt und das arithmetische Mittel des jeweiligen abhängigen Merkmals dieser Nachbarn in die Analyse integriert. Wir schätzen damit folgendes Modell:

$$
\ln (Y)=\boldsymbol{X} \boldsymbol{\beta}+\rho \boldsymbol{W} Y+\varepsilon
$$

mit den abhängigen Variablen BIP pro Kopf, Arbeitslosenrate, Wahlbeteiligung und Kriminalitätsrate (wobei der natürliche Logarithmus nur für das BIP verwendet wird). $X$ stellt die 
Matrix der unabhängigen Merkmale dar und $W$ ist eine binäre Matrix, die den Wert $w_{\mathrm{ij}}=1$ annimmt, wenn $j$ der Nachbarn des Landkreises $i$ ist. Die Anzahl der Nachbarn wird mit $k$ beschrieben. Somit ist $W Y$ der Vektor des arithmetischen Mittels für die Merkmale der angrenzenden Landkreise.

$W y_{i}=\sum_{j=1}^{k} y_{j}\left(w_{i j} / \sum_{j=1}^{k} w_{i j}\right)$

Insgesamt gehen in unsere Analysen 397 Landkreise und kreisfreie Städte ein (siehe Tabelle 3). ${ }^{5}$ Unser erstes Modell bezieht sich auf den natürlichen Logarithmus des Bruttoinlandsprodukts pro Kopf. Die Analyse zeigt, dass die Anzahl der Vereine in einem Landkreis einen statistisch signifikanten Zusammenhang mit dem Bruttoinlandsprodukt aufweist. Mit jedem zusätzlichen Verein pro tausend Einwohner lässt sich ein um 2,4 Prozent höheres Bruttoinlandsprodukt in den Kreisen beobachten. Das Bruttoinlandsprodukt hängt den Ergebnissen zufolge mit einer ganzen Reihe von weiteren Faktoren zusammen. So ist die Abiturientenquote positiv mit der Wirtschaftsleistung korreliert. Ein höheres BIP pro Kopf liegt auch in kreisfreien Städten im Vergleich zu Landkreisen vor und ist in Gebieten mit höherer Arbeitslosenquote geringer. Ein höherer Anteil des Dienstleistungssektors geht dagegen mit einer geringeren Bruttowertschöpfung einher. Das Bruttoinlandsprodukt der Landkreise wird, wie der Spatial-Lag-Effekt zeigt, nicht statistisch signifikant von der Höhe des durchschnittlichen Bruttoinlandsprodukts der angrenzenden Nachbarkreise beeinflusst. Die Vereinsdichte weist im Unterschied zum Bruttoinlandsprodukt dagegen keinen Zusammenhang mit der Arbeitslosenquote, der Wahlbeteiligung oder der Kriminalitätsrate der Regionen auf. Auch für die letzteren Modelle wurden wieder alle sinnvollen und verfügbaren Kontrollvariablen berücksichtigt. Die Modelle zeigen unter anderem, dass für die Arbeitslosenrate, die Wahlbeteiligung und die Kriminalitätsrate Autokorrelationen zwischen den Kreisen vorliegen.

Um die Robustheit dieser Ergebnisse zu testen, haben wir eine ganze Reihe von Variationen in den Analysen durchgeführt, die hier der Einfachheit halber nicht separat ausgewiesen werden. So wurden alternativ zur Gesamtzahl der Vereine alle vier Modelle auch mit der Anzahl an Vereinen in den drei Gruppen (1) Umwelt/Natur, Kultur und Sport, (2) Politik/Wirtschaft, Interessen und Soziales und (3) Freizeit geschätzt. Die Gesamtkriminalitätsrate wurde außerdem in sieben Straftatengruppen unterteilt (Körperverletzung, Diebstahl aus Wohnungen und Fahrzeugen, Sachbeschädigungen, Rauschgiftdelikte und Straßenkriminalität) und jeweils separat geschätzt. Des Weiteren haben wir eine Ausreißerkontrolle vorgenommen und die Anzahl der Fälle auf 98 Prozent (bzw. 95 und 90 Prozent) reduziert. Zusätzlich wurden die Ergebnisse auf Multikollinearität geprüft. Weil Landkreise eine relativ engräumige Einheit bilden und Einzugsgebiete über diese administrativen Grenzen hinweg bestehen, wurden die Vereinsdaten zudem für die 96 Raumordnungsregionen Deutschlands zusammengefasst und als Grundlage der Analysen verwendet. Keine dieser Variationen hat zu wesentlichen Änderungen der Ergebnisse geführt.

Statt den OLS-Modellen mit Spatial-Lag haben wir zum Vergleich auch einfache OLSRegressionen berechnet. Hierbei ergaben sich keine Differenzen der Ergebnisse. In der Literatur wird darüber hinaus die Anwendung von Maximum-Likelihood Verfahren empfohlen, weil diese bei Spatial-Lag Modellen zu konsistenteren Schätzungen führen (vgl. Franzese und

5 Für 31 Landkreise und kreisfreie Städte sind keine separaten Angaben zur Anzahl an Vereinen verfügbar, sondern nur die Gesamtzahl der Vereine der jeweiligen kreisfreien Stadt und des umliegenden Landkreises. Diese 30 Fälle wurden daher zu 15 Regionen zusammengefasst und durch eine DummyVariable (Mischtyp) gekennzeichnet. 
Hays 2007). Auch diese Variation führt in unserem Fall zu ähnlichen Ergebnissen. Allerdings schwächt sich der geschätzte Effekt der Vereine auf das BIP leicht auf 2,2\% ab. Wegen dieser lediglich geringfügigen Veränderung haben wir uns für die leichter verständliche Darstellung der Spatial OLS-Regression in Tabelle 3 entschieden.

Tabelle 3: OLS-Regression zur wirtschaftlichen und sozialen Prosperität von Landkreisen in Deutschland unter Berücksichtigung von Spatial-Lag-Effekten

\begin{tabular}{|c|c|c|c|c|}
\hline & $\begin{array}{c}\text { (1) } \\
\text { Brutto- } \\
\text { inlands- } \\
\text { produkt }^{\mathrm{a}}\end{array}$ & $\begin{array}{c}(2) \\
\text { Arbeits- } \\
\text { losenquote }\end{array}$ & $\begin{array}{c}\text { (3) } \\
\text { Wahl- } \\
\text { beteiligung }^{\mathrm{c}}\end{array}$ & $\begin{array}{c}\text { (4) } \\
\text { Krite }^{\mathrm{d}}\end{array}$ \\
\hline \multirow{3}{*}{ Konstante } & $4.349^{* * *}$ & -12.454 & $61.107^{* * *}$ & 64.947 \\
\hline & $(0.848)$ & $(7.517)$ & $(11.251)$ & $(68.951)$ \\
\hline & \multicolumn{4}{|c|}{$\begin{array}{c}\text { Unter Berücksichtigung von Dummy-Variablen für die } \\
\text { Bundesländer (siehe Tabelle A2) }\end{array}$} \\
\hline \multirow[t]{2}{*}{ Stadt $>100.000$ Einwohner } & 0.002 & -0.249 & $2.056^{* * *}$ & -5.215 \\
\hline & $(0.044)$ & $(0.341)$ & $(0.537)$ & (3.516) \\
\hline \multirow[t]{2}{*}{ kreisfreie Stadt } & $0.240^{* * *}$ & $1.372^{* * *}$ & $-3.415^{* * *}$ & $21.255^{* * *}$ \\
\hline & $(0.055)$ & $(0.407)$ & $(0.537)$ & $(4.338)$ \\
\hline \multirow{2}{*}{ Mischtyp } & 0.066 & $0.807^{*}$ & 0.794 & $68.055^{* * *}$ \\
\hline & $(0.037)$ & $(0.323)$ & $(0.473)$ & $(8.644)$ \\
\hline \multirow[t]{2}{*}{ Grenze mit Ausland } & -0.016 & 0.131 & $-1.068^{* *}$ & $5.208^{* *}$ \\
\hline & $(0.021)$ & $(0.179)$ & $(0.410)$ & $(1.863)$ \\
\hline \multirow[t]{2}{*}{ Bevölkerungsdichte } & -0.001 & $0.089^{* *}$ & $0.108^{* *}$ & 0.592 \\
\hline & $(0.003)$ & $(0.030)$ & $(0.037)$ & $(0.377)$ \\
\hline \multirow[t]{2}{*}{ Ausländeranteil } & $0.021^{* * *}$ & 0.006 & -0.034 & 0.050 \\
\hline & $(0.005)$ & $(0.030)$ & $(0.060)$ & $(0.319)$ \\
\hline \multirow[t]{2}{*}{ Anteil der 25 bis 65 Jährigen } & -0.002 & -0.061 & 0.188 & 0.054 \\
\hline & $(0.011)$ & $(0.091)$ & $(0.145)$ & $(0.794)$ \\
\hline \multirow[t]{2}{*}{ Anteil der $65+$ Jährigen } & -0.003 & $0.357^{* * * *}$ & $0.223^{*}$ & $-1.386^{*}$ \\
\hline & $(0.007)$ & $(0.065)$ & $(0.102)$ & $(0.604)$ \\
\hline \multirow[t]{2}{*}{ Total Sex Ratio } & -0.868 & $12.349^{* *}$ & -4.235 & -53.549 \\
\hline & $(0.588)$ & $(4.005)$ & $(5.994)$ & $(45.934)$ \\
\hline \multirow[t]{2}{*}{ Schulabgänger mit Allgemeiner Hochschulreife } & $0.012^{* * *}$ & -0.014 & $0.112^{* * *}$ & -0.020 \\
\hline & $(0.003)$ & $(0.014)$ & $(0.024)$ & $(0.135)$ \\
\hline \multirow[t]{2}{*}{ Anteil des Dienstleistungssektors } & $-0.013^{* * *}$ & -0.021 & $0.086^{* * *}$ & $0.384^{* *}$ \\
\hline & $(0.002)$ & $(0.012)$ & $(0.018)$ & $(0.137)$ \\
\hline \multirow[t]{2}{*}{ Anteil der Hilfeempfänger staatl. Leistungen } & $0.170^{* * *}$ & $3.435^{* * *}$ & $-2.865^{* * *}$ & $15.649^{* * *}$ \\
\hline & $(0.048)$ & $(0.355)$ & $(0.571)$ & $(4.274)$ \\
\hline \multirow[t]{2}{*}{ Arbeitslosenquote } & $-0.022^{* * *}$ & & $-0.652^{* * *}$ & $1.181^{*}$ \\
\hline & $(0.005)$ & & $(0.075)$ & $(0.536)$ \\
\hline \multirow[t]{2}{*}{ Bruttoinlandsprodukt ${ }^{\mathrm{b}}$} & & $-0.033^{* *}$ & -0.021 & $0.458^{* *}$ \\
\hline & & $(0.012)$ & $(0.016)$ & $(0.140)$ \\
\hline \multirow[t]{2}{*}{ Vereine pro 1.000 Einwohner } & $0.024^{* * *}$ & 0.052 & -0.057 & 0.206 \\
\hline & $(0.006)$ & $(0.042)$ & $(0.069)$ & $(0.488)$ \\
\hline \multirow[t]{2}{*}{ Spatial-Lag Ln Bruttoinlandsprodukt } & -0.020 & & & \\
\hline & $(0.019)$ & & & \\
\hline \multirow[t]{2}{*}{ Spatial-Lag Arbeitslosenquote } & & $0.308^{* * *}$ & & \\
\hline & & $(0.068)$ & & \\
\hline \multirow[t]{2}{*}{ Spatial-Lag Wahlbeteiligung } & & & $0.037^{* *}$ & \\
\hline & & & $(0.012)$ & \\
\hline \multirow[t]{2}{*}{ Spatial-Lag Kriminalitätsrate } & & & & $0.106^{*}$ \\
\hline & & & & $(0.052)$ \\
\hline $\mathrm{N}$ & 397 & 397 & 397 & 397 \\
\hline $\mathrm{R}^{2}$ & 0.77 & 0.89 & 0.80 & 0.84 \\
\hline
\end{tabular}

Anmerkung: Clustered standard errors in Klammern; ${ }^{*} p<0,05,{ }^{* *} p<0,01,{ }^{* * *} p<0,001 ;{ }^{\text {a }}$ Logarithmiertes reales Bruttoinlandsprodukt der Kreise pro Einwohner in tausend Euro; ${ }^{b}$ Reales Bruttoinlandsprodukt der 
Kreise pro Einwohner in tausend Euro; ${ }^{\mathrm{c}}$ Wahlbeteiligung an der Bundestagswahl; ${ }^{\mathrm{d}}$ Häufigkeit der Straftaten pro 1.000 Einwohner. Diese Angaben beziehen alle auf das Jahr 2009.

\section{Zusammenfassung und Diskussion}

Eine Analyse mit den Vereinsdaten zeigt, dass die Anzahl an Vereinen einen statistisch signifikanten und starken Zusammenhang mit dem Bruttoinlandsprodukt von Landkreisen und kreisfreien Städten aufweist. Dementsprechend geht jeder zusätzliche Verein pro 1.000 Einwohner mit einem höheren Bruttoinlandsprodukt von etwa 2,4 Prozent einher. Dieses Ergebnis bestätigt die Annahme, dass Vereine zur Bildung von Sozialkapital beitragen, was sich wiederum günstig auf die wirtschaftlichen Aktivitäten in Regionen auswirkt. Keine statistisch signifikanten Zusammenhänge konnten dagegen für die Arbeitslosenquote, die Wahlbeteiligung oder die Kriminalitätsrate gefunden werden. Dies besagt allerdings nicht, dass es diese Zusammenhänge nicht gibt, sondern könnte darauf hinweisen, dass die bloße Anzahl an Vereinen ein zu grober Indikator für die Messung des Sozialkapitals in Regionen ist.

Die vorgestellten Daten und Analysen haben gegenüber bestehenden Studien eine ganze Reihe von Vorteilen, aber auch einige Nachteile, die bei der Interpretation der Ergebnisse berücksichtigt werden müssen. Ein Vorteil unserer Analysen liegt darin, dass wir ausschließlich prozessproduzierte Daten verwenden, was sicherlich eine sinnvolle Ergänzung zu Analysen mit reaktiven Datenquellen darstellt. Darüber hinaus haben wir eine vergleichsweise aufwendige und vollständige Zählung der Vereine in Deutschland vorgenommen. Diese Daten erlauben es, 397 Regionen in einem Land zum Gegenstand der Untersuchung machen zu können. Viele vergleichbare Untersuchungen müssen sich dagegen auf relativ geringe Fallzahlen beschränken, die sich darüber hinaus in unterschiedlichen Ländern befinden. Wegen der politischen und kulturellen Unterschiede zwischen Gesellschaften sind die zu kontrollierenden Faktoren deshalb zahlreicher und geeignete Kontrollvariablen teilweise nicht oder nur unzureichend vorhanden.

Die Vereinsdaten weisen aber auch einige Probleme auf. Ein Nachteil ist sicherlich, dass wir für jede Region nur die Anzahl an Vereinen kennen und leider nichts über die Größe der Vereine, die Anzahl ihrer Mitglieder oder das Aktivitätsniveau wissen. Auch über die Art der Aktivität haben wir nur wenige Informationen. Putnam (2000) weist zum Beispiel darauf hin, dass Vereine einschließenden (bridging) oder ausschließenden (bonding) Charakter haben können. In der Literatur werden beide Typen auch als Putnam- und Olson-Vereine bezeichnet. Positive Effekte auf die wirtschaftliche und soziale Prosperität werden vor allem vom ersten Vereinstyp erwartet. Vereine, die besonderen Wert auf Exklusivität und spezielle Interessenvertretung einer sehr spezifischen Klientel legen, können dem Gemeinwohl auch abträglich sein. Die fehlenden Befunde in Bezug auf die Arbeitslosenquote, die Wahlbeteiligung oder die Kriminalitätsrate können damit auch durch die fehlende Genauigkeit der Daten über Vereine begründet sein. Zusätzlich haben wir es bei der Messung der Anzahl an Vereinen mit Registerdaten zu tun. Es ist gut möglich, dass die Vereinsregister bei den Amtsgerichten unterschiedlich sorgfältig geführt werden und damit eine mehr oder weniger große Anzahl an „Karteileichen" enthalten. Dieser Umstand würde natürlich die Schätzung verzerren. Eine Verbesserung ließe sich hier nur durch eine umfassende und repräsentative Vereinsbefragung herstellen, die Aussagen über die Zuverlässigkeit der Register sowie über die Art und Struktur der Vereine zulässt.

Ein weiterer wichtiger Nachteil ist darin zu sehen, dass wir keine kausalen Aussagen treffen können. Unsere Analysen zeigen lediglich, dass die Anzahl an Vereinen auch unter Kontrolle wichtiger anderer Einflussgrößen einen starken Zusammenhang mit dem Bruttoinlandsprodukt von Regionen aufweist. Putnams Hypothese unterstellt, dass Vereine die höhere Wirtschafts- 
leistung verursachen. Diese Aussage zur Richtung ist mit den uns zur Verfügung stehenden Daten aber nicht überprüfbar. Die unabhängige Variable „Vereinsdichte“ bezieht sich zwar auf das Jahr 2008 und die Messung des BIP pro Kopf auf das Jahr 2009. Aber theoretisch kann der Vereinsbestand des Jahres 2008 durch das BIP der Vorjahre verursacht worden sein und die BIP-Zahlen aus früheren Jahren korrelieren hoch mit denjenigen des Jahres 2009. Deswegen sind unsere Ergebnisse auch mit der Aussage kompatibel, dass erst ein höherer Wohlstand in einer Region zu einer erhöhten Vereinsgründung führt. Zur Prüfung der Kausalitätsannahme wären Paneldaten wünschenswert, die natürlich für die Anzahl an Vereinen in Regionen nicht vorliegen. Eine weitere Möglichkeit zur Überprüfung der Kausalitätsannahme wären sogenannte 2SLS-Modelle, bei denen mit Hilfe von Instrumentalvariablen zunächst die Anzahl der Vereine pro Region geschätzt werden und dann die durch das Modell prognostizierten Vereine im zweiten Schritt als erklärende Variable verwendet werden. Auf diese Weise ließe sich der durch das Bruttoinlandsprodukt erklärte Anteil an der Vereinszahl heraus rechnen. Allerdings sind hier keine geeigneten Instrumente zur Schätzung der Vereinszahlen für die Regionen vorhanden. Die vorgestellten Analysen stellen damit nur einen ersten Schritt dar, um die Funktion und den Beitrag von Vereinen statistisch zu untersuchen. Wünschenswert wäre die Berücksichtigung weiterer Information zu den Vereinen, die detaillierte Analysen ermöglichen würde. Ein solches Projekt erfordert eine umfangreiche Datenerhebung und bleibt der zukünftigen Forschung vorbehalten.

\section{Literatur}

Enquete-Kommission Zukunft des bürgerschaftlichen Engagements Deutscher Bundestag (Hrsg.) (2002 a): Bürgerschaftliches Engagement und Erwerbsarbeit, Band 9, Opladen.

Enquete-Kommission Zukunft des bürgerschaftlichen Engagements Deutscher Bundestag (Hrsg.), (2002 b): Bürgerschaftliches Engagement: Auf dem Weg in eine zukunftsfähige Bürgergesellschaft, Band 4, Opladen.

Almond, G.A. / Verba, S. (1963): The Civic Culture: Political Attitudes and Democracy in Five Nations, Princeton / NJ.

Alscher, M. / D. Dathe / E. Priller / R. Speth (2009): Bericht zur Lage und zu den Perspektiven des bürgerschaftlichen Engagements in Deutschland. Bundesministerium für Familie, Senioren, Frauen und Jugend (Hrsg.), Berlin: Wissenschaftszentrum Berlin für Sozialforschung.

Anders, G. (2005): Soziologische Sportvereinsforschung in der Bundesrepublik Deutschland, in: T. Alkemeyer / B. Rigauer / G. Sobiech (Hrsg.), Organisationsentwicklungen und De-Institutionalisierungsprozesse im Sport, Schorndorf, S. 99-122.

Anselin, L. (1988): Spatial Econometrics: Methods and Models, Dordrecht.

Bauer, J. / S. Braun (2001): Sportvereinsforschung in Deutschland, in: Sportwissenschaft 31, S. 199-212.

Beugelsdijk, S. / T. van Schaik (2005): Social Capital and Growth in European Regions: An Empirical Test, in: European Journal of Political Economy 21, S. 301-324.

Braun, J. / H. Klages (2009): Freiwilliges Engagement in Deutschland. Freiwilligensurvey 1999. Ergebnisse der Repräsentativerhebung zu Ehrenamt, Freiwilligenarbeit und bürgerschaftlichem Engagement, Band 2, Wiesbaden.

Breuer, C. / P. Wicker (2010): Sportvereine in Deutschland. Sportentwicklungsbericht 2009/2010 - Analyse zur Situation der Sportvereine in Deutschland, Köln.

Buonanno, P. / D. Montolio / P. Vanin (2009): Does Social Capital Reduce Crime?, in: Journal of Law \& Economics 52, S. 145-170.

Chi, G. / J. Zhu (2008): Spatial Regression Models for Demographic Analysis, in: Population Research and Policy Review 27, S. 17-42.

Coleman, J.S. (1990): Foundations of Social Theory, Cambridge. 
Emrich, E. / W. Pitsch / V. Papathanassiou (2001): Die Sportvereine. Ein Versuch auf empirischer Grundlage, Schorndorf.

Erlinghagen, M. (2000): Arbeitslosigkeit und Ehrenamtliche Tätigkeit im Zeitverlauf. Eine Längsschnittanalyse der westdeutschen Stichprobe des Sozio-oekonomischen Panels (SOEP) für die Jahre 1992 und 1996, in: Kölner Zeitschrift für Soziologie und Sozialpsychologie 52, S. 291-310.

Franzen, A. / Botzen, K. (2009): Die Vereinsstruktur Deutschlands, in: H.K. Anheier / N. Spengler (Hrsg.), Auf dem Weg zu einem Informationssystem Zivilgesellschaft, Essen, S. 52-59.

Franzen, A. / S. Pointner (2007): Sozialkapital: Konzeptualisierungen und Messungen, in: A. Franzen / M. Freitag (Hrsg.), Sozialkapital. Grundlagen und Anwendungen, Sonderheft 47 der Kölner Zeitschrift für Soziologie und Sozialpsychologie, Wiesbaden, S. 66-90.

Franzen, A. / D. Hangartner (2006): Social Networks and Labour Market Outcomes: The Non-Monetary Benefits of Social Capital, in: European Sociological Review 22, S. 353-368.

Franzese, R. J. / J.C. Hays (2007): Spatial Econometric Models of Cross-Sectional Interdependence in Political Science Panel and Time-Series-Cross-Section Data, in: Political Analysis 15, S. 140-164.

Freitag, M. (2000): Soziales Kapital und Arbeitslosigkeit. Eine empirische Analyse zu den Schweizer Kantonen, in: Zeitschrift für Soziologie 29, S. 186-201.

Freitag, M. / R. Traunmüller (2008): Sozialkapitalwelten in Deutschland. Soziale Netzwerke, Vertrauen und Reziprozitätsnormen im subnationalen Vergleich, in: Zeitschrift für vergleichende Politikwissenschaft 2, S. 221-256.

Fukuyama, F. (1995): Trust: The Social Virtues and the Creation of Prosperity, New York.

Gensicke, T. / S. Picot / S. Geiss (2006): Freiwilliges Engagement in Deutschland 1999 - 2004: Ergebnisse der repräsentativen Trenderhebung zu Ehrenamt, Freiwilligenarbeit und bürgerschaftlichem Engagement, Wiesbaden.

Gensicke, T. / T. Olk / D. Reim / J. Schmithals / H.L. Dienel (2009): Entwicklung der Zivilgesellschaft in Ostdeutschland. Quantitative und qualitative Befunde, Wiesbaden.

Granovetter, M.S. (1973): The Strength of Weak Ties, in: American Journal of Sociology 78, S. 1360-1380.

Granovetter, M.S. (1974): Getting a Job: A Study of Contacts and Careers, Cambridge.

Halpern, D. (2005): Social Capital, Cambridge.

Happes, W. (2008): Vereinsstatistik 2008. Konstanz: V\&M Service GmbH, abrufbar unter: http:// www.npo-info.de/vereinsstatistik/, letztes Abrufdatum: 20.10.2009.

Heinemann K. / M. Schubert (1994): Der Sportverein. Ergebnisse einer repräsentativen Untersuchung, Schorndorf.

Horch, H.D. (1992): Ressourcen und Oligarchie. Eine empirische Überprüfung, in: Kölner Zeitschrift für Soziologie und Sozialpsychologie 44, S. 99-115.

Jungbauer-Gans, M. (2002): Schwindet das Soziale Kapital?, in: Soziale Welt 53, S. 189-208.

Knack, S. / P. Keefer (1997): Does Social Capital Have an Economic Payoff? A Cross-Country Investigation, in: Quarterly Journal of Economics 112, S. 1251-1288.

Künemund, H. / J. Schupp (2007): Konjunkturen des Ehrenamts - Diskurs und Empirie, in: M. Erlinghagen / K. Hank (Hrsg.), Produktives Altern und informelle Arbeit in modernen Gesellschaften, Wiesbaden, S. 145-163.

Kunz, V. / O.W. Gabriel (2000): Soziale Integration und politische Partizipation. Das Konzept des Sozialkapitals - Ein brauchbarer Ansatz zur Erklärung politischer Partizipation?, in: U. Druwe / S. Kühnel / V. Kunz (Hrsg.), Kontext, Akteur und strategische Interaktion, Opladen, S. 47-74.

Lee, M.R. (2008): Civic Community in the Hinterland: Toward a Theory of Rural Social Structure and Violence, in: Criminology 46, S. 447-478.

LeSage, J. / R.K. Pace (2009): Introduction to Spatial Econometrics, Boca Raton. 
Lippl, B. (2007): Soziales Engagement und politische Partizipation in Europa, in: A. Franzen / M. Freitag (Hrsg.), Sozialkapital. Grundlagen und Anwendungen, Sonderheft 47 der Kölner Zeitschrift für Soziologie und Sozialpsychologie, Wiesbaden, S. 420-449.

Maloney, W.A. / J.W. van Deth / S. Roßteutscher (2008): Civic Orientations: Does Associational Type Matter?, in: Political Studies 56, S. 261-287.

Messner, S.F. / E.P. Baumer / R. Rosenfeld (2004): Dimensions of Social Capital and Rates of Criminal Homicide, in: American Sociological Review 69, S. 882-903.

Offe, C. / Fuchs, S. (2001): Schwund des Sozialkapitals? Der Fall Deutschland, in: R.D. Putnam (Hrsg.), Gesellschaft und Gemeinsinn. Sozialkapital im internationalen Vergleich, Gütersloh, S. 417-514.

Picot, S. (Hrsg.) (2001): Frauen und Männer, Jugend, Senioren, Sport, in: Freiwilliges Engagement in Deutschland - Freiwilligensurvey 1999 - Ergebnisse der Repräsentativerhebung zu Ehrenamt, Freiwilligenarbeit und bürgerschaftlichem Engagement, Band 3, Wiesbaden.

Priller, E. (2009): Der Bericht zur Lage und zu den Perspektiven des bürgerschaftlichen Engagements in Deutschland - Erfahrungen, Erkenntnisse und Herausforderungen, in: H.K. Anheier / N. Spengler (Hrsg.), Auf dem Weg zu einem Informationssystem Zivilgesellschaft, Essen, S. 23-28.

Priller, E. / J. Sommerfeld (2009): Wer spendet in Deutschland? Eine sozialstrukturelle Analyse, in: E. Priller / J. Sommerfeld (Hrsg.), Spenden in Deutschland, Berlin, S. 167-200.

Prognos/Generali (2009): Engagementatlas 2009: Daten. Hintergründe. Volkswirtschaftlicher Nutzen, Aachen.

Putnam, R.D. (1993): Making Democracy Work: Civic Traditions in Modern Italy, Princeton / NJ.

Putnam, R.D. (2000): Bowling Alone, New York.

Roth, R. (2001): Besonderheiten des bürgerschaftlichen Engagements in den neuen Bundesländern, in: Aus Politik und Zeitgeschichte B 39-40, S. 15-22.

Sampson, R.J. / S.W. Raudenbush / F. Earls (1997): Neighborhoods and Violent Crime: A Multilevel Study of Collective Efficacy, in: Science 277, S. 918-924.

Stadelmann-Steffen, I. / M. Freitag (2007): Der ökonomische Wert sozialer Beziehungen. Eine empirische Analyse zum Verhältnis von Vertrauen, sozialen Netzwerken und wirtschaftlichem Wachstum im interkulturellen Vergleich, in: A. Franzen / M. Freitag (Hrsg.), Sozialkapital. Grundlagen und Anwendungen, Sonderheft 47 der Kölner Zeitschrift für Soziologie und Sozialpsychologie, Wiesbaden, S. 294-320.

Strauß, S. (2009): Ehrenamt in Deutschland und Großbritannien - Sprungbrett zurück auf den Arbeitsmarkt?, in: Kölner Zeitschrift für Soziologie und Sozialpsychologie 61, S. 647-670.

Timm, W. (1979): Sportvereine in der Bundesrepublik Deutschland. Teil II: Organisations-, Angebotsund Finanzstruktur, Schorndorf.

Tocqueville, A. de [1840] (1976): Über die Demokratie in Amerika, Stuttgart.

Tolbert, C. / T. Lyson / M. Irwin (1998): Local Capitalism, Civic Engagement, and Socioeconomic WellBeing, in: Social Forces 77, S. 401-428.

Uhlendorff, A. (2004): Der Einfluss von Persönlichkeitseigenschaften und sozialen Ressourcen auf die Arbeitslosigkeitsdauer, in: Kölner Zeitschrift für Soziologie und Sozialpsychologie 56, S. 279-303.

van Deth, J.W. (2001): Soziale und politische Beteiligung: Alternativen, Ergänzungen oder Zwillinge?, in: A. Koch / M. Wasmer / P. Schmidt (Hrsg.), Politische Partizipation in der Bundesrepublik Deutschland: Empirische Befunde und theoretische Erklärungen, Opladen, S. 195-219.

von Rosenbladt, B. (Hrsg.) (2009): Gesamtbericht, in: Freiwilliges Engagement in Deutschland. Freiwilligensurvey 1999. Ergebnisse der Repräsentativerhebung zu Ehrenamt, Freiwilligenarbeit und bürgerschaftlichem Engagement, Band 1, Wiesbaden.

Voss, T. (2007): Netzwerke als soziales Kapital im Arbeitsmarkt, in: A. Franzen / M. Freitag (Hrsg.), Sozialkapital. Grundlagen und Anwendungen, Sonderheft 47 der Kölner Zeitschrift für Soziologie und Sozialpsychologie, Wiesbaden, S. 321-342. 
Ward, M.D. / K.S. Gleditsch (2008): Spatial Regression Models, Los Angeles.

Weber, M. (1924): Rede auf dem deutschen Soziologentag in Frankfurt 1910, in: M. Weber (Hrsg.), Gesammelte Aufsätze zur Soziologie und Sozialpolitik. Tübingen: J.C.B. Mohr, S. 431-449.

Whiteley, P. (2000): Economic Growth and Social Capital, in: Political Studies 48, S. 443-466.

Zimmer, A. (2007): Vereine - Zivilgesellschaft konkret, Wiesbaden.

Zimmer, A. / A. Bugari / G. Krötz (1992): Vereinslandschaften im Vergleich - Kassel, München, Zürich, in: A. Zimmer (Hrsg.), Vereine heute - zwischen Tradition und Innovation, Basel, S. 170-205.

Prof. Dr. Axel Franzen,

Katrin Botzen,

Universität Bern, Institut für Soziologie,

Lerchenweg 36,

CH-3012 Bern,

axel.franzen@soz.unibe.ch,

katrin.botzen@soz.unibe.ch 


\section{Anhang}

Tabelle A1: Deskriptive Tabelle der abhängigen und unabhängigen Variablen

\begin{tabular}{|c|c|c|c|c|c|c|c|}
\hline Variable & Quelle & $\begin{array}{l}\text { Ein- } \\
\text { heit }\end{array}$ & Min & Max & $\square$ & SD & $\mathrm{N}$ \\
\hline $\begin{array}{l}\text { Reales Bruttoinlandsprodukt der Kreise pro } \\
\text { Einwohner in tsd Euro }^{\mathrm{a}}\end{array}$ & $\begin{array}{l}\text { Stat. Bundes- und } \\
\text { Landesämter }\end{array}$ & $\mathrm{R}$ & 15,06 & 91,86 & 31,84 & 11,69 & 397 \\
\hline $\begin{array}{l}\text { Log. Bruttoinlandsprodukt der Kreise pro } \\
\text { Einwohner in tsd Euro }\end{array}$ & ebd. & $\mathrm{R}$ & 2,71 & 4,52 & 3,41 & 0,31 & 397 \\
\hline Arbeitslosenquote $^{\mathrm{b}}$ & $\begin{array}{l}\text { Statistisches Bun- } \\
\text { desamt }\end{array}$ & $\%$ & 2,50 & 20,10 & 8,77 & 3,87 & 397 \\
\hline $\begin{array}{l}\text { Wahlbeteiligung an der Bundestagswahl } \\
2009\end{array}$ & ebd. & $\%$ & 56,6 & 80 & 70,2 & 4,63 & 397 \\
\hline $\begin{array}{l}\text { Kriminalitätsrate: } \\
\text { Straftaten pro } 1.000 \text { Einwohner }\end{array}$ & $\begin{array}{l}\text { Bundeskriminal- } \\
\text { amt }\end{array}$ & $\mathrm{R}$ & 25,85 & 209,3 & 68,66 & 31,55 & 397 \\
\hline $\begin{array}{l}\text { Dummy Grenze: Landkreis mit Grenze zum } \\
\text { Ausland }=1\end{array}$ & eigene Recherche & & 0 & 1 & 0,16 & 0,36 & 397 \\
\hline $\begin{array}{l}\text { Dummy Stadt mit mehr als } 100.000 \text { Einwoh- } \\
\text { nern }=1\end{array}$ & ebd. & & 0 & 1 & 0,16 & 0,37 & 397 \\
\hline Dummy: kreisfreie Stadt $=1$ & ebd. & & 0 & 1 & 0,24 & 0,43 & 397 \\
\hline $\begin{array}{l}\text { Dummy Mischtyp: Kreisfreie Stadt und } \\
\text { Landkreis }=1\end{array}$ & ebd. & & 0 & 1 & 0,05 & 0,22 & 397 \\
\hline $\begin{array}{l}\text { Total Sex Ratio } \\
\text { (Männer zu Frauen) }\end{array}$ & $\begin{array}{l}\text { Statistisches Bun- } \\
\text { desamt }\end{array}$ & $\mathrm{R}$ & 0,87 & 1,02 & 0,96 & 0,03 & 397 \\
\hline Dichte Einwohner pro $100 \mathrm{~km}^{2}$ & ebd. & $\mathrm{R}$ & 0,38 & 42,82 & 5,00 & 6,70 & 397 \\
\hline $\begin{array}{l}\text { Schulabgänger mit Allgemeiner Hochschul- } \\
\text { reife }\end{array}$ & ebd. & $\%$ & 6,86 & 64,23 & 29,55 & 9,92 & 397 \\
\hline Ausländeranteil & ebd. & $\%$ & 0,75 & 24,86 & 7,07 & 4,39 & 397 \\
\hline Bevölkerungsanteil der 25 bis 65 Jährigen & ebd. & $\%$ & 50,36 & 59,99 & 54,11 & 1,56 & 397 \\
\hline Bevölkerungsanteil der $65+$ Jährigen & ebd. & $\%$ & 15,19 & 27,96 & 21,08 & 2,26 & 397 \\
\hline $\begin{array}{l}\text { Anteil des Dienstleistungssektors an der } \\
\text { Bruttowertschöpfung }\end{array}$ & ebd. & $\%$ & 22,70 & 74,90 & 50,15 & 8,47 & 397 \\
\hline Anteil der Empfänger staatl. Leistungen & ebd. & $\%$ & 0,28 & 2,48 & 0,99 & 0,40 & 397 \\
\hline $\begin{array}{l}\text { Gesamtzahl der Vereine } \\
\text { pro } 1.000 \text { Einwohner }\end{array}$ & eigene Erhebung & $\mathrm{R}$ & 1 & 17,34 & 6,86 & 1,94 & 397 \\
\hline $\begin{array}{l}\text { Natur- und Umweltvereine } \\
\text { pro } 1.000 \text { Einwohner }\end{array}$ & ebd. & $\mathrm{R}$ & 0 & 0,32 & 0,09 & 0,05 & 397 \\
\hline $\begin{array}{l}\text { Kulturvereine } \\
\text { pro } 1.000 \text { Einwohner }\end{array}$ & ebd. & $\mathrm{R}$ & 0 & 1,97 & 0,34 & 0,24 & 397 \\
\hline $\begin{array}{l}\text { Sportvereine } \\
\text { pro } 1.000 \text { Einwohner }\end{array}$ & ebd. & $\mathrm{R}$ & 0,20 & 5,92 & 1,22 & 0,69 & 397 \\
\hline $\begin{array}{l}\text { Sozial- und Wohlfahrtsvereine } \\
\text { pro } 1.000 \text { Einwohner }\end{array}$ & ebd. & $\mathrm{R}$ & 0,05 & 3,64 & 1,23 & 0,43 & 397 \\
\hline $\begin{array}{l}\text { Politik- und Wirtschaftsvereine } \\
\text { pro 1.000 Einwohner }\end{array}$ & ebd. & $\mathrm{R}$ & 0,05 & 5,12 & 0,95 & 0,55 & 397 \\
\hline $\begin{array}{l}\text { Interessensvereine } \\
\text { pro } 1.000 \text { Einwohner }\end{array}$ & ebd. & $\mathrm{R}$ & 0,05 & 1,69 & 0,53 & 0,24 & 397 \\
\hline $\begin{array}{l}\text { Freizeitvereine } \\
\text { pro } 1.000 \text { Einwohner }\end{array}$ & ebd. & $\mathrm{R}$ & 0,39 & 6,15 & 2,49 & 0,84 & 397 \\
\hline
\end{tabular}

Anmerkung: Sofern nicht anders angegeben, beziehen sich alle Angaben auf das Jahr 2009; ${ }^{a}$ Kaufkraftbereinigt in Bezug auf die Bundesländer. ${ }^{b}$ Die Arbeitslosenquote ist berechnet nach der Definition der Bundesagentur für Arbeit. 
Tabelle A2: OLS-Regression zur wirtschaftlichen und sozialen Prosperität von Landkreisen in Deutschland unter Berücksichtigung von Spatial-Lag-Effekten (zu Tabelle 3)

\begin{tabular}{|c|c|c|c|c|}
\hline & $\begin{array}{l}\text { (1) } \\
\text { Brutto- } \\
\text { inlands- } \\
\text { produkt }^{\text {a }}\end{array}$ & $\begin{array}{c}(2) \\
\text { Arbeits- } \\
\text { losenquote }\end{array}$ & $\begin{array}{c}\text { (3) } \\
\text { Wahlbetei- } \\
\text { ligung }^{\mathrm{c}}\end{array}$ & $\begin{array}{c}(4) \\
\text { Kriminali- }^{\text {tätsrate }}\end{array}$ \\
\hline \multirow[t]{3}{*}{ Konstante } & $4.349^{* * *}$ & -12.454 & $61.107^{* * *}$ & 64.947 \\
\hline & $(0.848)$ & $(7.517)$ & $(11.251)$ & $(68.951)$ \\
\hline & \multicolumn{4}{|c|}{$\begin{array}{l}\text { Unter Berücksichtigung derselben Kovariaten } \\
\text { wie in Tabelle } 3\end{array}$} \\
\hline Schleswig-Holstein & $\begin{array}{r}0.119^{*} \\
(0.050)\end{array}$ & $\begin{array}{l}-0.782 \\
(0.409)\end{array}$ & $\begin{array}{l}1.870^{* *} \\
(0.565)\end{array}$ & $\begin{array}{l}16.644^{* * *} \\
(4.907)\end{array}$ \\
\hline Niedersachsen & $\begin{array}{c}0.050 \\
(0.033)\end{array}$ & $\begin{array}{l}-0.381 \\
(0.276)\end{array}$ & $\begin{array}{l}1.418^{* * *} \\
(0.393)\end{array}$ & $\begin{array}{c}6.659 \\
(4.123)\end{array}$ \\
\hline Hessen & $\begin{array}{l}0.228^{* * *} \\
(0.047)\end{array}$ & $\begin{array}{c}0.071 \\
(0.333)\end{array}$ & $\begin{array}{c}0.231 \\
(0.486)\end{array}$ & $\begin{array}{l}-5.639 \\
(3.937)\end{array}$ \\
\hline Rheinland-Pfalz & $\begin{array}{l}-0.085^{*} \\
(0.042)\end{array}$ & $\begin{array}{l}-0.646 \\
(0.361)\end{array}$ & $\begin{array}{l}-1.636^{* * *} \\
(0.482)\end{array}$ & $\begin{array}{l}10.341^{* *} \\
(3.778)\end{array}$ \\
\hline Baden-Württemberg & $\begin{array}{l}0.130^{* *} \\
(0.041)\end{array}$ & $\begin{array}{l}-0.090 \\
(0.382)\end{array}$ & $\begin{array}{l}-2.100^{* * *} \\
(0.503)\end{array}$ & $\begin{array}{l}-1.278 \\
(4.702)\end{array}$ \\
\hline Bayern & $\begin{array}{l}0.238^{* * *} \\
(0.047)\end{array}$ & $\begin{array}{l}-0.643 \\
(0.374)\end{array}$ & $\begin{array}{l}-2.979^{* * *} \\
(0.531)\end{array}$ & $\begin{array}{l}-8.584 \\
(4.502)\end{array}$ \\
\hline Saarland & $\begin{array}{l}-0.208^{* *} \\
(0.073)\end{array}$ & $\begin{array}{l}-1.152^{*} \\
(0.507)\end{array}$ & $\begin{array}{c}0.367 \\
(0.729)\end{array}$ & $\begin{array}{c}1.187 \\
(4.856)\end{array}$ \\
\hline Brandenburg & $\begin{array}{l}-0.062 \\
(0.099)\end{array}$ & $\begin{array}{l}4.168^{* * *} \\
(0.932)\end{array}$ & $\begin{array}{l}-5.697^{* * *} \\
(0.935)\end{array}$ & $\begin{array}{l}18.363^{*} \\
(7.345)\end{array}$ \\
\hline Mecklenburg-Vorpommern & $\begin{array}{l}-0.002 \\
(0.069)\end{array}$ & $\begin{array}{l}3.959^{* * * *} \\
(0.800)\end{array}$ & $\begin{array}{l}-7.490^{* * *} \\
(1.160)\end{array}$ & $\begin{array}{l}18.716^{*} \\
(9.468)\end{array}$ \\
\hline Sachsen & $\begin{array}{c}0.046 \\
(0.069)\end{array}$ & $\begin{array}{l}4.043^{* * *} \\
(0.667)\end{array}$ & $\begin{array}{l}-7.026^{* * *} \\
(0.870)\end{array}$ & $\begin{array}{c}6.971 \\
(6.164)\end{array}$ \\
\hline Sachsen-Anhalt & $\begin{array}{c}0.070 \\
(0.058)\end{array}$ & $\begin{array}{l}3.703^{* * *} \\
(0.702)\end{array}$ & $\begin{array}{l}-9.017^{* * *} \\
(0.943)\end{array}$ & $\begin{array}{c}22.095^{* * *} \\
(6.326)\end{array}$ \\
\hline Thüringen & $\begin{array}{l}-0.076 \\
(0.066)\end{array}$ & $\begin{array}{l}3.290^{* * *} \\
(0.665)\end{array}$ & $\begin{array}{l}-8.235^{* * *} \\
(0.999)\end{array}$ & $\begin{array}{c}7.853 \\
(6.548) \\
\end{array}$ \\
\hline $\mathrm{N}$ & 397 & 397 & 397 & 397 \\
\hline $\mathrm{R}^{2}$ & 0.765 & 0.887 & 0.798 & 0.839 \\
\hline
\end{tabular}

Anmerkung: Clustered standard errors in Klammern; ${ }^{*} p<0,05,{ }^{* *} p<0,01,{ }^{* * *} p<0,001$; ${ }^{\text {a }}$ Logarithmiertes Bruttoinlandsprodukt der Kreise pro Einwohner in tausend Euro; ${ }^{b}$ Wahlbeteiligung an der Bundestagswahl 2009; ${ }^{\mathrm{c}}$ Häufigkeit der Straftaten pro 1.000 Einwohner. Diese Angaben beziehen sich alle auf das Jahr 2009. 